\title{
AN ANIMAL MODEL OF HUMAN ACID SPHINGOMYELINASE DEFICIENCY (NIEMANN- PICK DISEASE) AND THE STUDY OF ITS ENZYME REPLACEMENT
}

(The Japan Society of Human Genetics Award Lecture)

\author{
Teruo Kitagawa
}

Department of Pediatrics, Nihon University School of Medicine, Chiyoda-ku, Tokyo 101, Japan

In December 1975, two 10-old-mice, one female and one male, from a litter of the C57BL/KsJ inbred strain, which had been maintained in the Research Laboratories, Nippon Shinyaku Co., Ltd., since its transfer from the Jackson Laboratory in 1973 at $\mathbf{F}_{24}$, were found to be suffering from behavioral abnormalities (Miyawaki et al., 1982). These abnormalities were characterized by motor incoordination with coarse tremor of the body and the extremities. During the following 2 weeks the tremor became more pronounced. Finally the animals were unable to walk and their body weight decreased markedly due to difficulty in obtaining food and water.

Although these mice could not breed, mutant mice from the affected female have been bred by ovarian transplantation and maintained in the Research Laboratory. In 1982, we demonstrated a deficiency of sphingomyelinase activity and the accumulation of sphingomyelin in visceral organs of these mutant mice (Sakiyama et al., 1982). Hence, an animal model for Niemann-Pick disease has been developed.

This paper describes one series of our study on Niemann-Pick mice.

1. Identification of the Mice as an Analogue of Niemann-Pick Disease in Humans

Fresh tissue of mutant $\mathrm{C} 57 \mathrm{BL} / \mathrm{KsJ}$ strain mice with a progressive neurologic manifestation and hepatosplenomegaly were removed quickly after sacrifice, frozen and stored at $-20^{\circ} \mathrm{C}$ for several weeks prior to being analyzed for lipid or enzyme. Lipid analysis was carried out by thin-layer chromatography on HPTLC 60 silicagel plates. A chloroform-methanol $(2: 1)$ extract of tissues was prepared according to the method described by Folch et al. (1957). Plates were one-dimensionally chromatographed using chloroform-methanol-water $(60: 35: 8)$ as a solvent, and sphingomyelin was visualized by vaporing with Dittmer reagent and quantitated by color

Received April 20, 1987

Presented on the occasion of receiving the Japan Society of Human Genetics Award at the Annual Meeting of the Japan Society of Human Genetics, Tokyo, November 6-8, 1986. 
scanning. Quantitative determinations of cholesterol and its esters were carried out according to Zak's method (Zak, 1957) both with and without digitonin precipitation on the total lipid extract. Ganglioside content was assayed by the colorimetric method with a resorcinol reagent. Sphingomyelin obtained from the liver of affected mice was purified by Folch's partition method followed by column chromatography on Iatrobeads, and fatty-acid composition of sphingomyelin was determined by gasliquid chromatography.

The tissues were thawed and homogenized in distilled water containing $0.1 \%$ Triton X-100 $(20 \% \mathrm{w} / \mathrm{v})$ and centrifuged at $10,000 \times g$ for $20 \mathrm{~min}$. The supernatants were employed for enzyme assays. Sphingomyelinase activity was measured using the chromogenic artificial substrate 2- $N$-hexadecanoylamino-4-nitrophenylphosphorylcholine hydroxide according to the Gal method (Gal et al., 1975) and also by using [choline-methyl $-{ }^{14} \mathrm{C}$ ]sphingomyelin at $\mathrm{pH} 5.5$ in $1 \mathrm{~mol} / \mathrm{liter}$ acetate buffer solution according to the Kanfer method (Kanfer et al., 1966) with slight modification. Other lysosomal acid hydrolases were determined by fluorometric assay using 4-methylumbelliferone derivatives as substrates. Protein content was measured by the Lowry method (Lowry et al., 1951).

The quantities of cholesterol, sphingomyelin, and ganglioside in tissues are shown in Table 1. The accumulated cholesterol in the affected mice was almost $100 \%$ free cholesterol. A marked accumulation of cholesterol and sphingomyelin as well as a moderate excess of ganglioside was noted in the visceral organs of the affected mice, but an accumulation in the brain was not noted. The accumulated lipids, purified from the liver of affected mice, were identical to the standard sphingomyelin on infrared spectrophotometry.

The activities of sphingomyelinase, $\beta-N$-acetyl hexosaminidase [EC 3.2.1.52], $\beta$-glucuronidase [EC 3.2.1.31], $\beta$-galactosidase [EC 3.2.1.23], and $\beta$-glucosidase [EC 3.2.1.21] in the tissues are shown in Table 1. Sphingomyelinase activity measured by artificial substrate in the liver of affected mice was about $30 \%$ of controls, in the spleen about $50 \%$, and in the brain $70-80 \%$. Residual sphingomyelinase activity of the affected mice, assayed using natural substrate, was nearly $10 \%$ in the liver, about $40 \%$ in the spleen, and $70 \%$ in the brain. From a precise study of the $\mathrm{pH}$ profile and isoelectric focusing pattern of sphingomyelinase, acid sphingomyelinase was found to be deficient in the liver and spleen, but neutral sphingomyelinase was not deficient in the affected mice (Miyawaki et al., 1982; Sakiyama et al., 1982).

The results presented here clearly demonstrate that the affected mouse is an authentic model for Niemann-Pick disease in humans.

2. Isoelectric Focusing Patterns of Sphingomyelinase in Tissues from Normal and Niemann-Pick Mice

Niemann-Pick disease in humans has tentatively been classified into five different phenotypes, taking into account the age of onset, the progression of the clinical course, the degree of neurological involvement and visceral-organ manifestation, the 
Table 1. Lipid contents and enzyme activities in pooled tissues from affected and control mice.

\begin{tabular}{llrr}
\hline & Tissues & $\begin{array}{c}\text { Affected } \\
\text { mice }\end{array}$ & $\begin{array}{c}\text { Control } \\
\text { mice }\end{array}$ \\
\hline Lipid contents (mg/g wet tissue) & & & \\
Cholesterol & liver & 37.3 & 4.3 \\
& spleen & 26.9 & 2.7 \\
& brain & 24.2 & 20.0 \\
Sphingomyelin & liver & 16.9 & 3.9 \\
& spleen & 16.8 & 1.3 \\
& brain & 4.2 & 3.0 \\
Ganglioside & liver & 1.9 & 0.4 \\
& spleen & 0.5 & 0.2 \\
Enzyme activities (nmol/mg protein/h) & brain & 1.3 & 0.6 \\
Sphingomyelinase by artificial substrates & liver & 14.6 & 48.9 \\
& spleen & 8.2 & 19.2 \\
& brain & 77.6 & 108.1 \\
Sphingomyelinase by natural substrates & liver & 5.9 & 44.6 \\
& spleen & 20.5 & 53.2 \\
$\beta$-Hexosaminidase & brain & 108.5 & 153.6 \\
$\beta$-Glucuronidase & liver & 6,720 & 1,810 \\
$\beta$-Glucosidase & liver & 1,810 & 615 \\
\hline
\end{tabular}

${ }^{\text {a }}$ Measured by using artificial substrates.

activity of sphingomyelinase, and certain other criteria. The affected mouse in the present study was similar to both type $A$ and $C$ of Niemann-Pick disease because of the progression of neurologic manifestations in the clinical course.

An analogy to type $C$ was suggested in that there was a late onset of neuropathy. In the beginning of our study, chemical procedures failed to demonstrate any accumulation of sphingomyelin in CNS. There was also no marked sphingomyelinase deficiency in the whole brain homogenate, in spite of a progressive deterioration of brain function. In order to elucidate the biochemical cause of their neurological manifestations, an additional study on sphingomyelinase in brain and liver of Niemann-Pick mice was carried out by using isoelectric focusing (Vesterberg et al., 1966).

The isoelectric focusing patterns of sphingomyelinase in the liver and the brain from both a control and an affected mouse are illustrated in Fig. 1 (Tsuda et al., 1985). In the control liver, two major peaks of sphingomyelinase activity, A and 

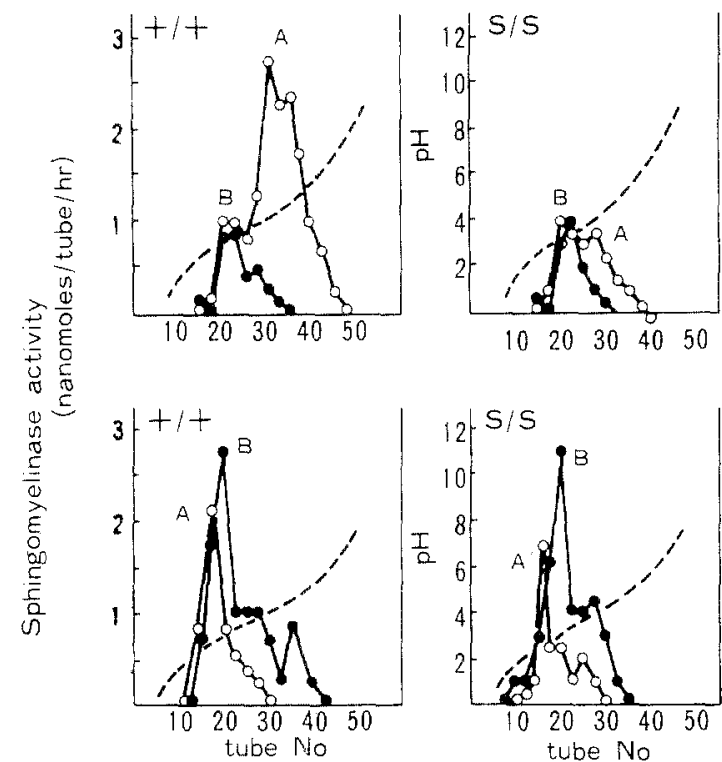

Fig. 1. Isoelectric focusing pattern of sphingomyelinase in mice tissue extracts. Enzyme activities are indicated on the left-hand vertical axis and tube numbers on the horizontal axis. The dotted line indicates the $\mathrm{pH}$ gradient. Upper panels: sphingomyelinases in liver. Lower panels: sphingomyelinases in brain. $+1+$ indicates control mice, and $\mathrm{s} / \mathrm{s}$ Niemann-Pick mice. indicates sphingomyelinase activity at $\mathrm{pH} 7.5$, and $\mathrm{O}$ sphingomyelinase activity at $\mathrm{pH}$ 5.5.

$\mathrm{B}$, are seen. Peak A, with a pI of 4.2 , indicates acid sphingomyelinase and peak $\mathrm{B}$ with a $\mathrm{pI}$ of 3.0 shows neutral sphingomyelinase. In the affected liver, peak B was quite similar to that of the control, whereas a significant decline of peak A was noticed.

In the control brain two major peaks of sphingomyelinase activity, $\mathrm{A}^{\prime}$ and $\mathrm{B}^{\prime}$, were also observed. However, in contrast to the liver, peak $B^{\prime}$ with a pI of 3.0, which indicated neutral sphingomyelinase, was the most prominent peak, and the pI of acid sphingomyelinase (peak $A^{\prime}$ ) was significantly different from that of the liver as shown in Fig. 1. In the affected brain, two peaks of sphingomyelinase activity were also seen. This pattern did not differ from the control brain.

This study using isoelectric focusing, failed to demonstrate a deficiency of acid sphingomyelinase in the mutant mouse. However, histological examination showed a loss of Purkinje cells in the cerebellum and a ballooning of retinal ganglion cells. Also electron microscopy disclosed clear membranous cytoplasmic bodies in retinal ganglion cells (Hagita et al., 1986).

On the basis of the above findings, we speculated that some kind of abnormality of sphingomyelinase in the affected brain must exist. 
3. Subcellular Localization of Acid Sphingomyelinase and Lipids in Normal and Niemann-Pick Mice

A study was performed on the subcellular localization of sphingomyelinase and lipids in brain and liver of control and affected mice. The procedures used were as follows. After sacrifice, the livers were taken out immediately and put into 9 volumes of ice-cold $0.25 \mathrm{~mol} /$ liter $^{-1}$ sucrose. Centrifugal fractionation was performed according to the Hogeboom procedure (Hogeboom, 1955). The brains were also similarly processed in $0.32 \mathrm{~mol} /$ liter $^{-1}$ sucrose (Clendenon and Allen, 1970). After getting a crude mitochondria-lysosome fraction, additional centrifugal fractionation was performed by the De Robertis method (De Robertis et al., 1962) which left us with three subfractionations. The lipid analysis, protein determination and sphingomyelinase activity of each individual fraction was determined in a similar fashion to the previous report (Sakiyama et al., 1982). Determiner PL (Kyowa Medix) and Cholesterol C-Test (Wako) were used for the quantitative determination of sphingomyelin and cholesterol.

In Fig. 2, we can see the subcellular localization of the content of sphingomyelin and cholesterol as well as acid sphingomyelinase activity in livers and brains (Sakiyama et al., 1987) given in order of their centrifugal fractionation. A morphological study using electron-microscopy supported the reliability of centrifugal fractionation for microsome and mitochondria-lysosome. The shape of the fractionized microsome in the control and the Niemann-Pick liver was similar. The shape of the mitochondria-lysosome in the Niemann-Pick liver was remarkably different from that of the control. Similar changes were noticed in the mitochondria and lysosome of Niemann-Pick brain, where the mitochondria and lysosome were ballooned and the membranous cytoplasmic bodies were accumulated (Fig. 3).

In the whole homogenate, acid sphingomyelinase activity of the affected liver and brain showed approximately $25 \%$ and $65 \%$ of the control activities, respectively (Sakiyama et al., 1982). However, the acid sphingomyelinase activity in the crude mitochondria-lysosomal fractions of affected Niemann-Pick liver and brain were reduced to approximately $10 \%$ and $20 \%$ of the normal control fraction. Additional centrifugal fractionation of the mitochondria-lysosome compartment clearly showed reduced activity of acid sphingomyelinase in pure mitochondria-lysosome fragments. The existence of these fragments was confirmed electronmicroscopically. Moreover, the accumulated sphingomyelin and cholesterol was observed in the crude-mitochondria-lysosomal and microsome fractions of the liver. The accumulation of lipids was remarkable in the upper part of the fractioned layer when compared to the lysosomal fraction (Fig. 2) (Sakiyama et al., 1987). In the microsome of the control and Niemann-Pick brains, the isoelectric focusing pattern and the activity of acid sphingomyelinase seemed almost identical. In the mitochondria-lysosomal fraction of Niemann-Pick brain, however, the activity of acid sphingomyelinase was reduced to only $10 \%$ of normal control, and without a different isoelectric focusing pattern (Fig. 4). 


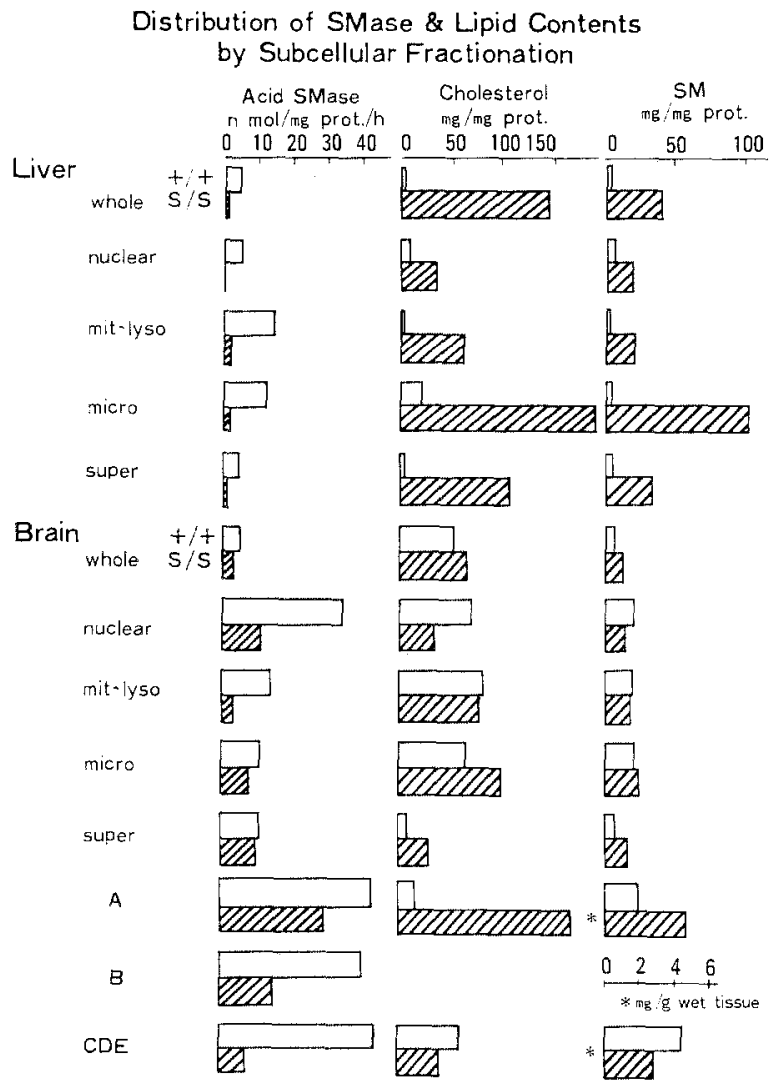

Fig. 2. Open bars indicate control and shaded bars Niemann-Pick mouse. Subcellular localization was determined by the method written in text. The lower graph indicates the additional centrifugal fractionations from crude mitochondria-lysosomal solution (brain): A denotes myelin and membrane fragment; B denotes small fragments of nerve endings containing synaptic vesicles, curved membranes and some myelin fragments; CDE denotes nerve endings containing synaptic vesicle, isolated nerve endings and free mitochondria and lysosomes.

The above findings suggest that reduced acid sphingomyelinase in the lysosome of the brain is probably responsible for the development of neurological manifestations in the affected mice. It may also effect the composition of the membrane in the lysosome of the brain as well as in the liver. It might also be responsible for the morphological change of the ballooning of mitochondria and lysosome, and the consequential escaping of the lipid from that compartment, which in turn increases the lipid content of the upper phase. Our findings strongly suggest that in fact, the Niemann-Pick mouse is very similar to the Niemann-Pick type A in humans, although the time of onset of the neurological manifestations is delayed. Because it has been reported that Niemann-Pick type C (McKusick 25720) in human shows no marked sphingomyelin accumulation and no significant sphingomyelinase defi- 
control

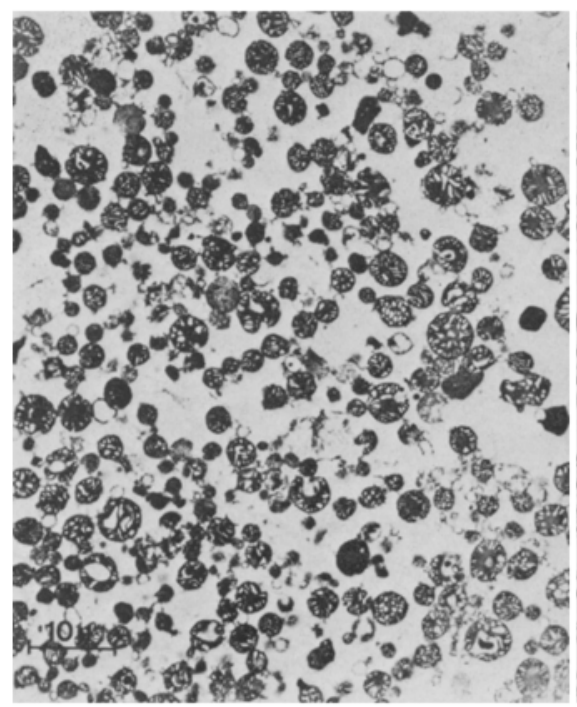

control

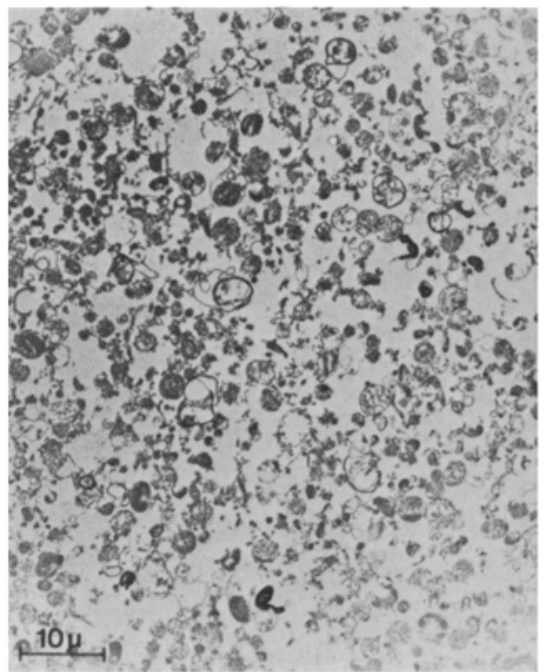

$\mathrm{spm} / \mathrm{spm}$

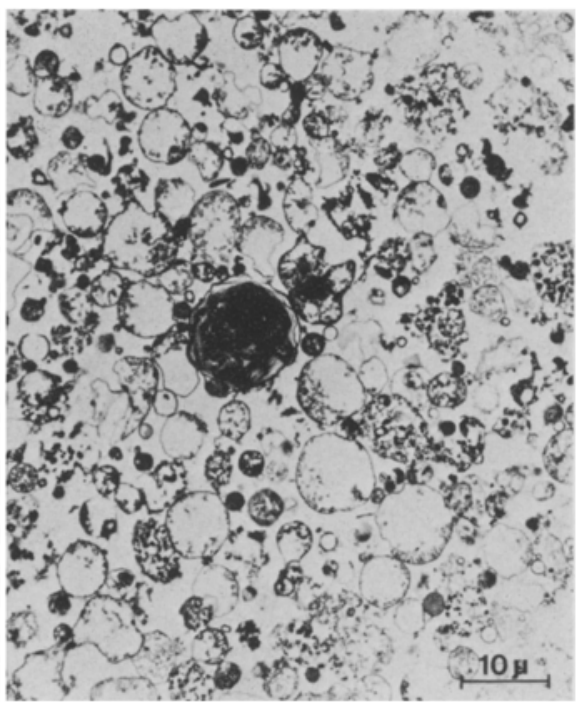

$\mathrm{spm} / \mathrm{spm}$

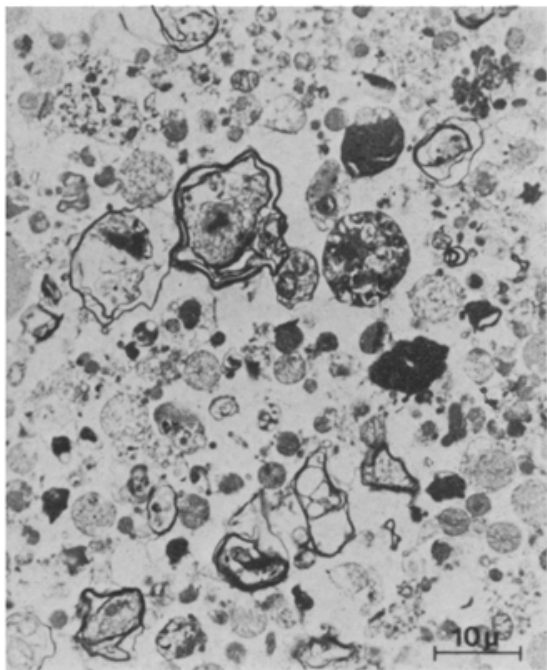

Fig. 3. Electron-microscopy of mitochondria-lysosomal fraction of liver (top) and brain (bottom) from inbred control mice (left) and from Niemann-Pick mice (right). Mitochondria-lysosome in Niemann-Pick liver and brain were ballooned and the membranous cytoplasmic bodies were accumulated.

ciency in brain homogenates, although there is neurological manifestations. In 1985, Pentchev et al. reported that the cultivated fibroblasts from Niemann-Pick disease type $\mathrm{C}$ showed a poor cholesterole sterification. Activity of cholesterol esterification of the fibroblasts from Niemann-Pick mouse is now under the study. 


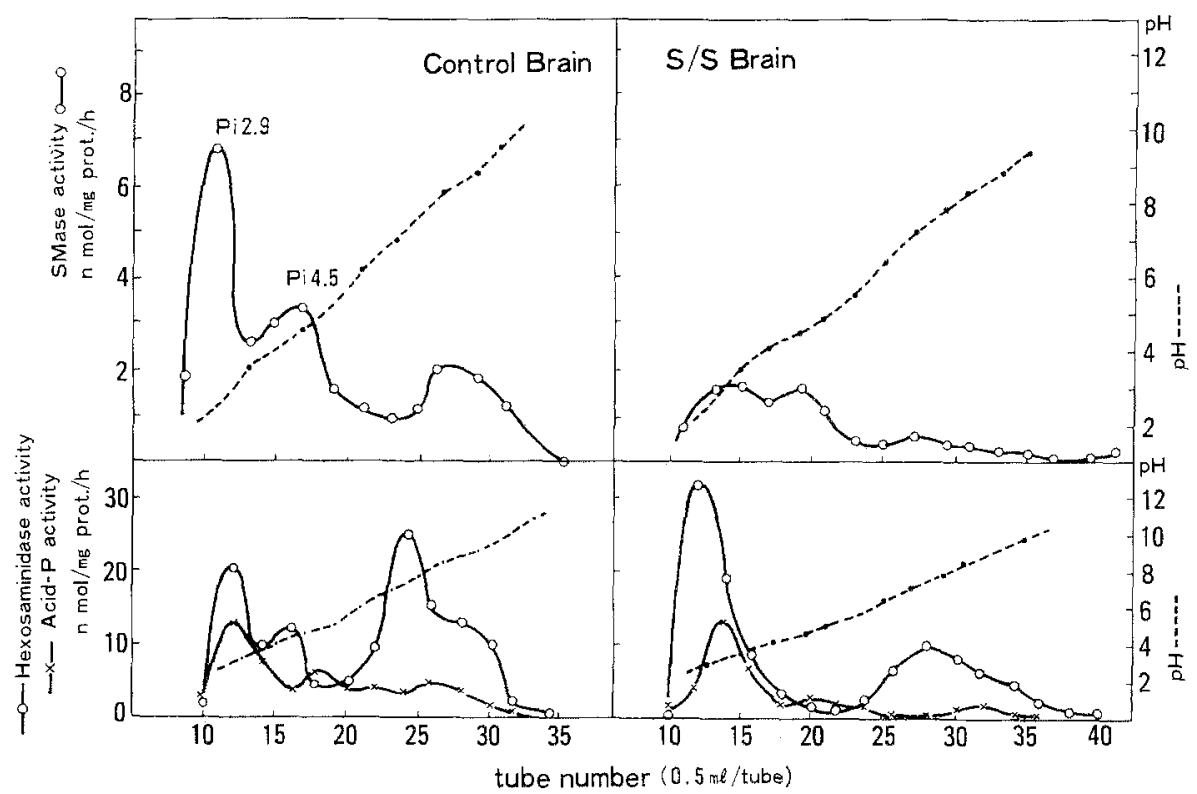

Fig. 4. Isoelectric focusing pattern of sphingomyelinase, $\beta$-hexosaminidase and acid phosphatase in crude mitochondria-lysosomal fraction from Niemann-Pick mice (right) and control inbred mice (left). Enzyme activities are indicated on the left-hand vertical axis and tube numbers on the horizontal axis. The dotted line indicates the $\mathrm{pH}$ gradient.

\section{Bone Marrow Transplantation for Niemann-Pick Mice}

Recently, some experimental therapies have been tried for the lysosomal storage disease such as Niemann-Pick disease, Hurler's disease and other diseases (Brady et al., 1980; Hobbs et al., 1981; Hobbs, 1985). Concerning Niemann-Pick disease, for example, a bone-marrow transplant could provide an adequate amount of sphingomyelinase to degrade accumulated sphingomyelin deposits and prevent further deposition, which should result in a clinical improvement of the disease. These necessary investigations should be first examined with appropriate animal models, because of the ethical and scientific limitations on human experimentation. Therefore, we tested the possibility that a bone marrow transplant for Niemann-Pick mice would result in a biochemical as well as clinical improvement of the disease (Sakiyama et al., 1983; 1985). Our procedure of bone marrow transplantation in mice is as follows.

The detection of splenomegaly by laparotomy was carried out to diagnose affected mice at 4 weeks of age. Bone marrow cells were collected from upper and lower leg bones of inbred mice and these were suspended in RPMI1640 (Gibco) containing $2 \%$ fetal calf serum and were sieved through Teflon mesh. The collected cells were washed and precipitated by centrifugation at $1,000 \mathrm{rpm}$ for $10 \mathrm{~min}$, and resuspended in the same medium. The cell count was adjusted to $2 \times 10^{7}$ in $0.2 \mathrm{ml}$ 
medium. All procedures were performed on ice under sterile conditions. This cell suspension $(0.2 \mathrm{ml})$ was injected into affected mice through the tail vein at the age of approximately 5 weeks. The mice were irradiated just prior to transplantation.

In the first experiment (Sakiyama et al., 1983), the Niemann-Pick mice that were irradiated with 900 rads survived only 2 days after the graft. The Niemann-Pick mice irradiated with 400,750 and 850 rad survived longer after transplantation, but their lifespans were not prolonged and the onset of neurological signs such as ataxia and body weight loss started at 7-8 weeks of age with death at 10-12 weeks of age, almost the same time as compared with non-grafted Niemann-Pick mice (Fig. 5). However, the accumulation of sphingomyelin in the bone marrow of an affected Niemann-Pick mouse was apparently reduced by the bone-marrow graft.

Body Weignt of Bone Marrow Transplanted Mice

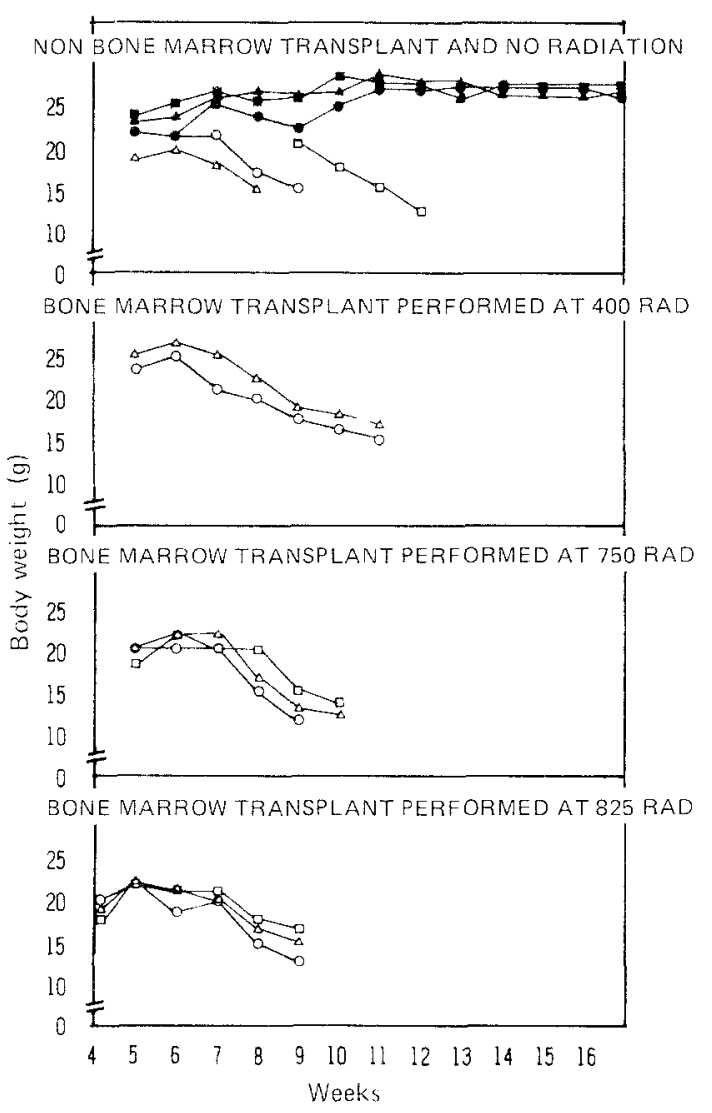

Fig. 5. The life spans and the body weight curves of Niemann-Pick mice. In the top graph, the closed marks indicate the control mice while the open marks denote the affected mice. The other graphs represent mice which had bone marrow transplants. 
This reduction was proved by histochemical staining. In addition, accumulation of sphingomyelin and cholesterol in spleen was remarkably decreased after the graft. The content of sphingomyelin and cholesterol in the liver was also slightly reduced. The mice irradiated with 400,750 and 850 rad all reacted similarly (Table 2).

In the second experiment (Sakiyama et al., 1985), two 5-week-old mice with Niemann-Pick disease were transplanted with normal mice bone marrow cells immediately after total body irradiation of 750 rads. These grafted Niemann-Pick mice were sacrificed 3 weeks later and tissues were removed for the biochemical and pathological studies.

Light microscopic examination of the spleen in grafted mutant mice revealed a dramatic decrease in accumulated lipid depositions, compared with the massive clusters of foamy cells in non-grafted Niemann-Pick mice (Fig. 6).

In the liver, lipid accumulation was only slightly reduced. The severe depletion of Purkinje cells in the cerebellum, characteristic of non-grafted Niemann-Pick mice, was about the same in the grafted Niemann-Pick mice.

The tissues from the liver and spleen were examined for their lipid content after extraction with chloroform-methanol $(2: 1 \mathrm{v} / \mathrm{v})$. Compared with the tissues of Niemann-Pick mice, the spleens of the grafted Niemann-Pick mice showed a $33 \%$ decrease in sphingomyelin content (still about twice as much as the normal controls), and a $50 \%$ decrease (still about three times the normal controls) in cholesterol content. The liver, however, showed only a slight decrease or no change in both cholesterol and sphingomyelin contents (Table 3).

These findings are comparable to the pathological changes and lipid content

Table 2. Amounts of total lipid, total cholesterol and sphingomyelin in liver and spleen of spm/spm mice with or without bone marrow transplantation.

\begin{tabular}{lcccc}
\hline & Total lipid & $\begin{array}{c}\text { Total cholesterol } \\
\text { (mg/g tissue) }\end{array}$ & Sphingomyelin \\
\hline Liver & & & & \\
non-grafted spm/spm & 1 & 292 & 45.0 & 14.7 \\
& 2 & 284 & 46.0 & 16.1 \\
$400 \mathrm{rad} \mathrm{spm} / \mathrm{spm}$ & & 132 & 51.1 & 10.6 \\
$825 \mathrm{rad} \mathrm{spm} / \mathrm{spm}$ & 1 & 132 & 52.1 & 11.1 \\
& 2 & 116 & 36.7 & 10.2 \\
Spleen & & & & 7.5 \\
non-grafted spm/spm & 1 & 108 & 29.3 & 8.1 \\
& 2 & 80 & 23.4 & 2.7 \\
$400 \mathrm{rad} \mathrm{spm} / \mathrm{spm}$ & & 42 & 12.5 & 2.9 \\
$750 \mathrm{rad} \mathrm{spm} / \mathrm{spm}$ & & 54 & 9.9 & 1.7 \\
$825 \mathrm{rad} \mathrm{spm} / \mathrm{spm}$ & 1 & 38 & 7.2 & 2.6 \\
\hline
\end{tabular}




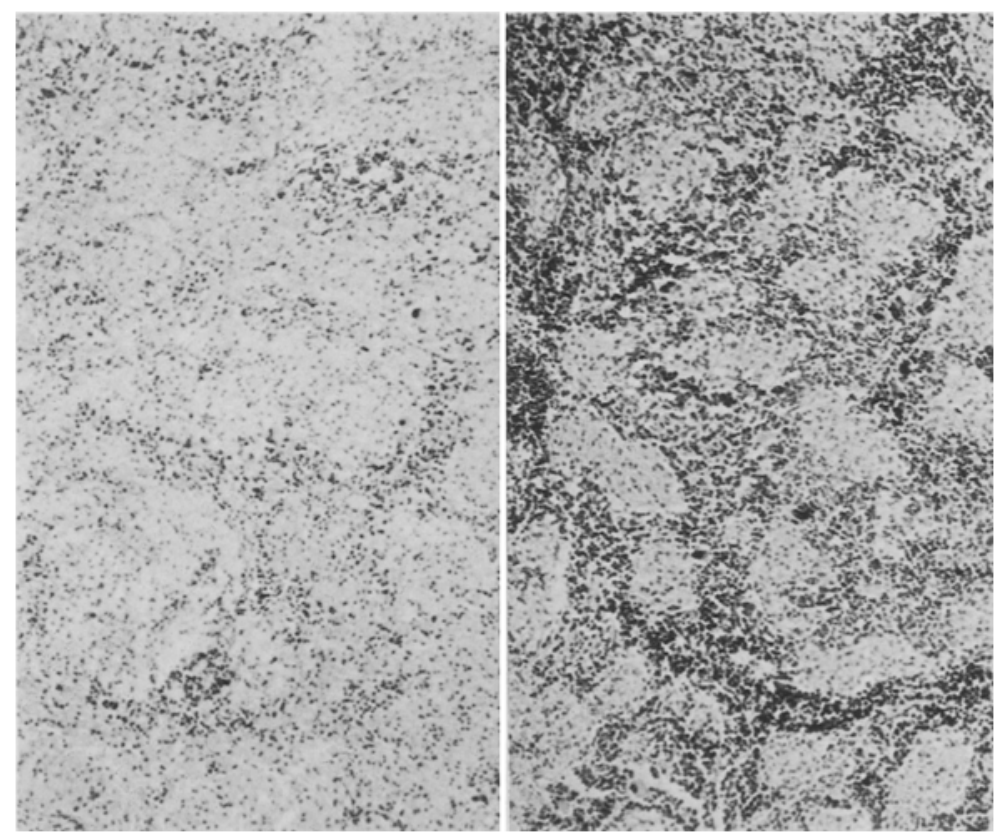

Fig. 6. Photograph of spleen from non-grafted Niemann-Pick mouse (left) and bonemarrow grafted Niemann-Pick mouse (right) at 8-week-old. There was marked infiltration of foamy cells in non-grafted mouse but they were reduced in grafted mouse.

of tissue samples. Liver from the grafted Niemann-Pick mice had about twice the sphingomyelinase activity (about $1 / 3$ of normal mice controls) and the spleen had a three-fold increase (almost the same as the normal controls) compared with nongrafted Niemann-Pick mice (Table 3). The above findings show that an increase of sphingomyelinase activity may be responsible for the decrease of lipid content in visceral tissues. On the other hand, the neurological involvement of the NiemannPick mice was not improved by the graft. This might be ascribed to the existence of blood-brain barriers which prohibit the leukocytes from entering the necessary area of the brain. Our result might suggest a limitation of the unqualified application of bone marrow transplants to patients with lysosomal storage disease showing neurological involvement. However, bone marrow transplants for the lysosomal storage diseases without neurological manifestation seems to be a successful treatment.

\section{Transplantation of Bone Marrow Cells from Niemann-Pick Mice into Inbred Normal Mice}

Increase of sphingomyelinase activity and a decrease of the accumulated sphingomyelin in liver and spleen after transplantation of bone marrow cells from inbred normal mice into Niemann-Pick mice are supposed to be due to repopulation of 
Table 3. Sphingomyelin, cholesterol and acid sphingomyelinase activity in the livers and spleens of NP mice either 3 weeks after transplant or without bone marrow transplantation.

Lipid contents are expressed as milligram per gram wet tissue. Acid sphingomyelinase activities are expressed as nanomoles of substrate hydrolyzed per milligram of protein per hour. All results are the mean of duplicate experiments.

\begin{tabular}{lccc}
\hline & \multicolumn{2}{c}{ Lipid contents } & \\
\cline { 2 - 3 } & $\begin{array}{c}\text { Cholesterol } \\
\text { (mg/g wet tissue) }\end{array}$ & $\begin{array}{c}\text { Sphingomyelinase } \\
\text { (nmol/mg protein/h) }\end{array}$ \\
\hline Liver & \multicolumn{3}{c}{} \\
$\quad$ Normal control mice & 4.8 & 3.9 & 67.6 \\
NP & 43.2 & 15.9 & 10.7 \\
Grafted NP 1 & 25.0 & 16.5 & 19.5 \\
Grafted NP 2 & 22.8 & 11.0 & 23.7 \\
Spleen & & & 38.9 \\
Normal control mice & 4.1 & 1.7 & 12.0 \\
NP & 23.4 & 10.2 & 37.1 \\
Grafted NP 1 & 15.7 & 4.6 & 46.3 \\
Grafted NP 2 & 9.9 & 2.3 & \\
\hline
\end{tabular}

the reticuloendothelial and lymphohaematopoietic systems in the recipient tissues. However, the cellular transport and enzyme replacement strategies have not been well documented. In order to clarify how the grafted normal bone marrow cell corrects the abnormal lipid storage in viscera, we examined biochemical and pathological changes in bone marrow Niemann-Pick cells transplanted into inbred normal mice (Sakiyama et al., 1986).

Four inbred normal mice were given $750 \mathrm{rad}$ of whole body irradiation 4-5 hr prior to transplantation of bone marrow cells from Niemann-Pick mice.

One mouse died at 4 weeks after bone marrow transplantation, and another two were killed at 4 weeks and 28 weeks after the marrow graft. Another mouse which was marrow grafted similarly was killed at 7 weeks after bone marrow transplantation. At necropsy, the normal inbred mouse which died at 4 weeks after transplantation of bone marrow from Niemann-Pick mice showed only a few foamy mononuclear phagocytes which sparsely infiltrated along the sinusoid and vessels in the spleen. Similar findings were noted in the spleen of the normal inbred mouse which was killed at 4 weeks after transplantation. Niemann-Pick cells were found difffusedly with a few formations of clusters mainly in the red pulp of the spleen and around sinusoids of the liver in the mice which were killed at 7 and 28 weeks after transplantation. Foamy cell infiltration seemed to be scattered sparsely throughout the bloodstream. In the brain, however, foamy cells could not be found (Fig. 7).

Cholesterol and sphingomyelin contents increased at 7 weeks after transplanta- 


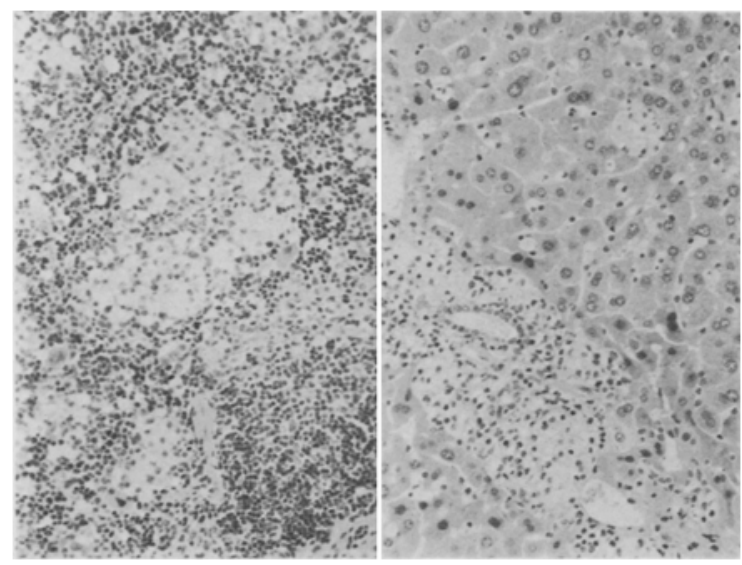

Fig. 7. Foamy cells are found diffusedly with formation of clusters mainly in the red pulp of the spleen (left) and around the sinusoid of the liver (right) in the $(+/+)$ mouse at 28 weeks after transplantation of bone marrow cells from Niemann-Pick mice ( $\times 200, \mathrm{H}$ and $\mathrm{E}$ staining).

tion. The contents of cholesterol and sphingomyelin in the liver were 9.2 and 5.6 $\mathrm{mg} / \mathrm{g}$ wet tissue, respectively, and in the spleen were 11.3 and $5.0 \mathrm{mg} / \mathrm{g}$ wet tissue, respectively. Compared with normal mice, these levels were almost twice as great in the liver and four times greater in the spleen. In the livers and spleens of the 4 and 28 week mice, the contents of accumulated lipids did not affect the biochemical analysis. They showed almost identical values to the control. Sphingomyelinase activities in these tissues at 4,7 and 28 weeks after bone marrow transplantation were similar to the values in the normal C57BL/KsJ mice, although there was a slight tendency to reduced activity in the spleen of the 7 week mouse (34.2 versus 51.5 and $40.2 \mathrm{nmol} / \mathrm{mg}$ protein $/ \mathrm{h}$ ). These biochemical findings seemed to be almost parallel to the morphological changes in normal recipient mice transplanted with Niemann-Pick bone marrow.

These findings strongly imply that host cells and transferred macrophages coexist but that the primary metabolic lesion resides in the transferred cells only and is not a consequence of the extracellular lesion influencing the environment of the normal cells. This suggests that the entry of lysosomal enzymes from cells into other cells may not play an important role in the biochemical and clinical ionprovement in transplant for lysosomal storage diseases. After the termination of the life span of the host cells, accumulated materials that escaped from the cells may be digested by transferred macrophage-monocyte system. It seems that the above mechanism may be responsible for the treatment of the lysosomal storage disease by bone marrow transplantation.

\section{Conclusion}

This series of our study revealed that the mutant mice with progressive neuro- 
logical manifestations and hepatosplenomegaly found in a strain of C57BL/KsJ mice is an animal model of Niemann-Pick disease type $\mathrm{A}$ in humans. This mutant mouse is one of the most suitable animal models for the research of lysosomal storage diseases. Niemann-Pick mice have provided us with valuable and attractive results on the pathological and biochemical investigation of the disease and on the experimental trials of bone marrow transplantation or other enzyme replacement therapy. Our results have encouraged us to apply bone marrow transplantation to patients with lysosomal storage diseases excluding patients with neurological involvement. It also might warn of unqualified application of bone marrow transplants to humans with lysosomal storage disease showing neurological storage.

Acknowledgments We thank Drs. T. Sakiyama, M. Owada and M. Tsuda for the biochemical studies, Mr. S. Miyawaki for the supply of mutant mice, Drs. M. Tadokoro and T. Shinagawa for the histopathological studies and Dr. Cecil Fox for helpful suggestions. This research was supported in part by a Grant-in-Aid for New Drug Development from the Ministry of Health and Welfare and by the Grant-in-Aid for Developmental Scientific Research, Inborn Errors of Metabolism from the Ministry of Education, Science and Culture (58480259, 57870069) of Japan.

\section{REFERENCES}

Brady, R.O., Barranger, J.A., Gal, A.E., Pentchev, P.G., and Furbish, F.S. 1980. Status of enzyme replacement therapy for Gaucher disease. In Enzyme Therapy in Genetic Diseases, Desnick, R.J. ed., Liss, New York, Vol. 2, pp. 361-368.

Clendenon, N.R. and Allen, N. 1970. Assay and subcellular localization of the arylsulfatase in rat brain. J. Neurochem. 17: 749-757.

De Robertis, E., De Iraldi, P., De Lores, G.R., and Salganicoff, L. 1962. Cholinergic and noncholinergic nerve endings in rat brain-I. J. Neurochem. 9: 23-35.

Folch, J., Lees, M., and Sloane-Stanley, G.H. 1957. A simple method for the isolation and purification of total lipids from animal tissue. J. Biol. Chem. 226: 497-509.

Gal, A.E., Brady, R.O., Hibbert, S.R., and Pentchov, P.G. 1975. A practical chromogenic procedure for the detection of homozygotes and heterozygous carriers of Niemann-Pick disease. N. Engl. J. Med. 293: 626-632.

Hagita, K., Shimada, H., Matsui, M., and Kitagawa, T. 1986. Basic study on ocular manifestation of Niemann-Pick disease. Part 1. Morphological study of the retina and choroid of "NiemannPick" mice. Acta Soc. Ophthalmol. Jpn. 90: 570-580.

Hobbs, J.R. 1985. Correction of 34 genetic diseases by displacement bone marrow transplantation. Plasma Ther. Transfus. Technol. 6: 221-246.

Hobbs, R.J., Hugh-Jones, K., Barrett, A.J., Byrom, N., Chambers, D., Henry, K., James, D.C.O., Lucas, C.F., Rogers, T.R., Benson, P.F., Tansley, L.R., Patrick, A.D., Mossman, J., and Young, E.P. 1981. Reversal of clinical features of Hurler's disease and biochemical improvement after treatment by bone marrow transplantation. Lancet 2: 709-712.

Hogeboom, G.H. 1955. Fractionation of cell components of animal tissues. In Methods in Enzymology, Colowick, S.P. and Kaplan, N.V. ed., Academic Press, New York, Vol. 1, pp. 16-19.

Kanfer, J.N., Young, O.M., Shapiro, D., and Brady, R.O. 1966. The metabolism of sphingomyelin. I. Purification and properties of a sphingomyelin-cleaving enzyme from rat liver tissue. J. Biol. Chem. 241 : 1081-1084.

Lowry, O.H., Rosebrough, N.J., Farr, A.L., and Randall, R.J. 1951. Protein measurement with 
the Folin phenol reagent. J. Biol. Chem. 193: 265-275.

Miyawaki, S., Mitsuoka, S., Sakiyama, T., and Kitagawa, T. 1982. Sphingomyelinosis; A new mutation in the mouse; a model of Niemann-Pick disease in humans. J. Hered. 73: 257-263.

Pentchev, P.G., Comly, M.C., Kruth, H.S., Vanier, M.T., Wenger, D.A., Patel, S., and Brady, R.O. 1985. A defect in cholesterol esterification in Niemann-Pick disease (type C) patients. Proc. Natl. Acad. Sci. USA 82: 8247-8251.

Sakiyama, T., Tsuda, M., Kitagawa, T., Fujita, R., and Miyawaki, S. 1982. A lysosomal storage disorder in mice; a model of Niemann-Pick disease. J. Inher. Metab. Dis. 5: 239-240.

Sakiyama, T., Tsuda, M., Owada, M., Joh, K., Miyawaki, S., and Kitagawa, T. 1983. Bone marrow transplantation for Niemann-Pick mice. Biochem. Biophys. Res. Commun. 113: 605-610.

Sakiyama, T., Tsuda, M., Owada, M., Nakabayashi, H., Miyawaki, S., and Kitagawa. T. 1985. Therapeutic bone marrow transplantation in Niemann-Pick mice. Acta Paediatr. Jpn. 27: 64 68.

Sakiyama, T., Tsuda, M., Owada, M., Kitagawa, T., Miyawaki, S., Shinagawa, T., and Tadokoro., M. 1986. Bone marrow transplantation in Niemann-Pick mice. J. Inher. Metab. Dis. 9: 305-308.

Sakiyama, T., Akashi, K., Akatsuka, A., Owada, M., Miyawaki, S., and Kitagawa, T. 1987. Subcellular localization of acid sphingomyelinase and lipid in Niemann-Pick mice. $J$. Inher. Metab. Dis. 10 : in press.

Tsuda, M., Owada, M., Kitagawa, T., and Miyawaki, S. 1985. Lack of acid sphingomyelinase in the mitochondrialysosome fraction of brain from Niemann-Pick mice. J. Inher. Metab. Dis. 8: 147-148.

Vesterberg, O. and Svensson, H. 1966. Isoelectric fractionation analysis, and characterization of ampholytes in natural pH gradients. Acta Chem. Scand. 20: 820-834.

Zak, B. 1957. Simple rapid microtechnic for serum total cholesterol. Am. J. Clin. Pathol. 27: 583-588. 